\title{
SUMS OF HERMITIAN SQUARES ON PSEUDOCONVEX BOUNDARIES
}

\author{
Mihai Putinar and Claus Scheiderer
}

\begin{abstract}
We give an abstract characterization of all real algebraic subvarieties of complex affine space on which every positive polynomial is a sum of hermitian squares, and we find obstructions to this phenomenon. As a consequence we construct a strictly pseudoconvex domain with smooth algebraic boundary on which there exists a degree two positive polynomial which is not a sum of hermitian squares, answering thus in the negative a question of John D'Angelo.
\end{abstract}

\section{Introduction}

We identify real affine space $\mathbb{R}^{2 d}$ of even dimension with complex affine space $\mathbb{C}^{d}$, with coordinates $(x, y)=\left(x_{1}, \ldots, x_{d}, y_{1}, \ldots, y_{d}\right) \in \mathbb{R}^{2 d}$ resp. $z=\left(z_{1}, \ldots, z_{d}\right) \in \mathbb{C}^{d}$, so that $z_{k}=x_{k}+i y_{k}(1 \leq k \leq d)$. The Euclidean norm is denoted

$$
\|z\|^{2}=\sum_{k=1}^{d}\left|z_{k}\right|^{2}=\sum_{k=1}^{d}\left(x_{k}^{2}+y_{k}^{2}\right) .
$$

Let $\mathbb{C}[z, w]$ be the polynomial ring in $2 d$ variables $z_{j}, w_{j}(j=1, \ldots, d)$. On $\mathbb{C}[z, w]$ we consider the $\mathbb{C} / \mathbb{R}$-involution $*$ given by $z_{j}^{*}=w_{j}(j=1, \ldots, d)$. We call $f=f(z, w)$ real if $f=f^{*}$, and we write $A$ for the ring of real (i.e., $*$-fixed) elements in $\mathbb{C}[z, w]$. Thus $A=\mathbb{R}[x, y]$ is the real polynomial ring in the $2 d$ variables $x=\frac{1}{2}(z+w)$ and $y=\frac{1}{2 i}(z-w)$. The $\mathbb{R}$-homomorphisms $A \rightarrow \mathbb{R}$ correspond to the points $\alpha \in \mathbb{C}^{d}$ via $(z, w) \mapsto(\alpha, \bar{\alpha})$, or alternatively, to the points $(\xi, \eta)$ in $\mathbb{R}^{2 d}$ via $(x, y) \mapsto(\xi, \eta)$.

We often identify a polynomial $f(z, w) \in \mathbb{C}[z, w]$ with its induced hermitian polynomial map $\mathbb{C}^{d} \rightarrow \mathbb{C}, z \mapsto f(z, \bar{z})$. This map commutes with complex conjugation if and only if $f=f^{*}$, i.e., $f$ is real. Conversely, if $h: \mathbb{C}^{d} \rightarrow \mathbb{C}, z \mapsto h(z, \bar{z})$ is an hermitian polynomial map, the polynomial $f(z, w) \in \mathbb{C}[z, w]$ with $h(z)=f(z, \bar{z})$ is called the polarization of $h$.

Let $I \subset A$ be an ideal, and let

$$
X:=V_{\mathbb{R}}(I)=\operatorname{Hom}_{\mathbb{R}}(A / I, \mathbb{R})=\left\{\alpha \in \mathbb{C}^{d}: \forall f \in I, f(\alpha, \bar{\alpha})=0\right\}
$$

be the real zero set of $I$. The elements of the quotient algebra $A / I$ can be considered as real polynomial functions on $X$. Let $\Sigma(A / I)^{2}$ denote the convex cone of sums of squares in $A / I$.

A real hermitian polynomial $f(z, \bar{z})$ is called an hermitian square if $f(z, \bar{z})=|p(z)|^{2}$ for some polynomial $p(z) \in \mathbb{C}[z]$. Let $\Sigma_{h} \subset A$ denote the convex cone of sums of hermitian squares, so $\Sigma_{h}$ consists of the finite sums $\sum_{\nu} f_{\nu}^{*} f_{\nu}$ with $f_{\nu} \in \mathbb{C}[z]$. Clearly, $\Sigma_{h} \subset \Sigma A^{2}$, and it is easy to see that this inclusion is proper. Given an ideal $I \subset A$

Received by the editors May 25, 2010. 
as above, we correspondingly write $\Sigma_{h}(A / I):=\left(\Sigma_{h}+I\right) / I$ for the cone of sums of hermitian squares restricted to $X$. There are nontrivial examples of ideals $I$ for which $\Sigma_{h}(A / I)$ contains every function in $A / I$ strictly positive on $X$. The most notable example is the (ideal of the) unit sphere in $\mathbb{C}^{d}$, in which case the observation goes back independently to Quillen [14], Athavale [4] and Catlin-D'Angelo [6], [7]. To be more specific, Quillen proved that a bi-homogeneous polynomial which is positive on the sphere is a sum of (homogeneous) hermitian squares of polynomials, Athavale proved that a commutative tuple $T=\left(T_{1}, \ldots, T_{n}\right)$ of Hilbert space operators satisfying the sphere equation $T_{1}^{*} T_{1}+\ldots+T_{n}^{*} T_{n}=I$ is subnormal, while Catlin and D'Angelo proved that every positive polynomial on the sphere is equal to a sum of hermitian squares of polynomials, when restricted to the sphere. The operator theory community was well aware a long time ago that Athavale's theorem is equivalent to Catlin and D'Angelo result, see for instance $[12,13]$.

The aim of the present note is to work towards a characterization of all ideals $I$ in $A$ with the property that $\Sigma_{h}(A / I)$ contains all polynomials strictly positive on $V_{\mathbb{R}}(I)$. In the case of closed pseudoconvex real hypersurfaces of $\mathbb{C}^{d}$, such a result was sought by complex analysts, after the resurrection of Quillen's theorem by Catlin and D'Angelo. See specifically the comments in [1] and the open problem raised by D'Angelo at the 2006 meeting "Complexity of mappings in CR geometry", American Institute of Mathematics, Palo Alto.

The link between a sum of hermitian squares restricted on the boundary of a domain $G \subset \mathbb{C}^{n}$ and proper polynomial maps (or even proper embeddings) of $G$ into the unit ball of a higher dimensional affine space comes naturally into discussion, see $[6,7]$. In this respect it is worth mentioning the early work of Løw; he proved in [10] that a continuous, positive function $f$ on a strictly pseudoconvex $C^{2}$-smooth boundary $\partial G$ can be decomposed as $f=\sum_{k=1}^{m}\left|g_{k}\right|^{2}$, where the functions $g_{k}$ are analytic in $G$ and continuous on $\bar{G}$. Also, Lempert $[8,9]$ has proved in the real analytic framework that a positive function on $\partial G$ is the sum of a series of hermitian squares of analytic functions in $G$ (which are real analytic on its closure). A conclusion of our note is that the polynomial framework is much more rigid, revealing a large gap between positive elements and sums of hermitian squares on strictly pseudoconvex boundaries or on more general real algebraic varieties. To measure this gap it is natural to consider duality and separating linear functionals, leading to the interpretation of sums of hermitian squares as polars to solutions of moment problems with real algebraic support. This can be done in the context of subnormality of tuples of commuting operators and positivity of non-commutative functional calculi. Some results in this direction will appear in a forthcoming article, together with more details about the real algebraic geometry observations sketched below.

\section{Semirings}

Let $B$ be an $\mathbb{R}$-algebra, and let $S$ be a subsemiring of $B$ with $\mathbb{R}_{+} \subset S$. Recall that $S$ is said to be archimedean (in $B$ ) if $\mathbb{R}+S=B$, that is, if for every $f \in B$ there exists a real number $c$ such that $c \pm f \in S$. If $B$ is generated by $x_{1}, \ldots, x_{n}$, then $S$ is archimedean if and only if there exist $c_{i} \in \mathbb{R}$ with $c_{i} \pm x_{i} \in S(i=1, \ldots, n)$. See ([11] Definition 5.4.1 [15] 1.5 and references there).

Recall that $A$ denotes the ring of real hermitian polynomials $f(z, \bar{z})$. 
Definition. Let $I$ be an ideal in $A$. We say that $\Sigma_{h}$ is archimedean modulo $I$ if the semiring $\Sigma_{h}+I$ is archimedean in $A$, or equivalently, if the semiring $\Sigma_{h}(A / I)$ is archimedean in $A / I$. have

By (a particular case of) the Representation Theorem ([11] Theorem 5.4.4), we

Theorem 2.1. Let I be an ideal in A. The following conditions on I are equivalent:

(i) $V_{\mathbb{R}}(I)$ is compact, and every $f \in A$ with $f>0$ on $V_{\mathbb{R}}(I)$ lies in $\Sigma_{h}+I$;

(ii) $\Sigma_{h}$ is archimedean modulo $I$.

(The Representation Theorem, in the version for semirings, asserts that (ii) implies (i). The opposite implication is obvious.)

We observe the following simple characterization of these ideals:

Proposition 2.2. Let $I$ be an ideal in $A$. Then $\Sigma_{h}$ is archimedean modulo $I$ if and only if I contains an hermitian polynomial of the form

$$
f(z, \bar{z})=c+\|z\|^{2}+\sum_{k=1}^{r}\left|q_{k}(z)\right|^{2}
$$

with $c \in \mathbb{R}$ and $q_{k}(z) \in \mathbb{C}[z](k=1, \ldots, r)$.

Proof. When $\Sigma_{h}$ is archimedean modulo $I$, there exists $c \in \mathbb{R}$ with $c-\|z\|^{2} \in \Sigma_{h}+I$, which implies the above condition. Conversely, if $-\left(c+\|z\|^{2}\right) \in \Sigma_{h}+I$ then $V_{\mathbb{R}}(I)$ is compact, and also

$$
(1-c) \pm 2 x_{j}=\left|z_{j} \pm 1\right|^{2}+\sum_{k \neq j}\left|z_{k}\right|^{2}-\left(c+\|z\|^{2}\right)
$$

and

$$
(1-c) \pm 2 y_{j}=\left|z_{j} \pm i\right|^{2}+\sum_{k \neq j}\left|z_{k}\right|^{2}-\left(c+\|z\|^{2}\right)
$$

lie in $\Sigma_{h}+I$, for $j=1, \ldots, d$. This implies that $\Sigma_{h}$ is archimedean modulo $I$.

This gives plenty of examples of ideals $I$ such that every polynomial strictly positive on $V_{\mathbb{R}}(I)$ is a hermitian sum of squares modulo $I$. In particular we have obtained in this way an algebraic proof and explanation of Quillen and Catlin-D'Angelo theorems:

Proposition 2.3. On a real hypersurface of $\mathbb{C}^{d}$ with equation

$$
\|z\|^{2}+\sum_{k=1}^{r}\left|q_{k}(z)\right|^{2}=M
$$

where $q_{k} \in \mathbb{C}[z]$ and $M>0$, every strictly positive polynomial is a sum of hermitian squares. 


\section{Obstructions}

The basic observation which leads to simple obstructions for having universal hermitian squares decompositions of positive polynomials along a real variety is that a positive semi-definite Hermitian form satisfies the Cauchy-Schwarz inequality. To better cast this observation we introduce a couple of definitions.

For $\alpha, \beta \in \mathbb{C}^{d}$ we consider the ideals

$$
\mathfrak{a}_{\alpha}:=\sum_{j=1}^{d}\left(z_{j}-\alpha_{j}\right), \quad \mathfrak{b}_{\beta}:=\sum_{j=1}^{d}\left(w_{j}-\beta_{j}\right)
$$

of $\mathbb{C}[z, w]$. We define

$$
J_{\alpha, \beta}:=\mathfrak{a}_{\alpha} \mathfrak{a}_{\beta}+\mathfrak{b}_{\bar{\alpha}} \mathfrak{b}_{\bar{\beta}}
$$

Writing $\mathfrak{m}_{\alpha, \beta}=\mathfrak{a}_{\alpha}+\mathfrak{b}_{\beta}$, the maximal ideal $\mathfrak{m}_{\alpha, \beta}$ of $\mathbb{C}[z, w]$ consists of all $f(z, w)$ with $f(\alpha, \beta)=0$. When $\alpha \neq \beta$, therefore,

$$
J_{\alpha, \beta}=\mathfrak{m}_{\alpha, \bar{\alpha}} \cap \mathfrak{m}_{\beta, \bar{\beta}} \cap \mathfrak{m}_{\alpha, \bar{\beta}} \cap \mathfrak{m}_{\beta, \bar{\alpha}}
$$

consists of all $p(z, w) \in \mathbb{C}[z, w]$ with

$$
p(\alpha, \bar{\alpha})=p(\beta, \bar{\beta})=p(\alpha, \bar{\beta})=p(\beta, \bar{\alpha})=0 .
$$

Similarly, $J_{\alpha, \alpha}$ consists of all $p(z, w) \in \mathbb{C}[z, w]$ with

$$
p(\alpha, \bar{\alpha})=\frac{\partial p}{\partial_{z_{j}}}(\alpha, \bar{\alpha})=\frac{\partial p}{\partial_{\bar{z}_{k}}}(\alpha, \bar{\alpha})=\frac{\partial^{2} p}{\partial_{z_{j}} \partial_{\bar{z}_{k}}}(\alpha, \bar{\alpha})=0
$$

for $j, k=1, \ldots, d$.

Proposition 3.1. Let $I \subset A$ be an ideal, and consider two distinct points $\alpha, \beta \in \mathbb{C}^{d}$. Assume that $I \subset J_{\alpha, \beta}$. Then there exists a polynomial $f \in A$ which is strictly positive on $V_{\mathbb{R}}(I)$ and for which $f \notin \Sigma_{h}^{2}+I$.

Proof. Since all real polynomials separate the points of $\mathbb{R}^{2 d}$, there exists $f \in A$ with $f>0$ on $V_{\mathbb{R}}(I)$ and such that the Cauchy-Schwarz inequality

$$
|f(\alpha, \bar{\beta})|^{2} \leq f(\alpha, \bar{\alpha}) f(\beta, \bar{\beta})
$$

fails. Indeed, we can choose $f=\epsilon+h^{2}$, where $h \in A$ satisfies $h(\alpha, \bar{\alpha})=0, h(\beta, \bar{\beta})=0$, $h(\alpha, \bar{\beta})=1$ and $\epsilon>0$ is small.

On the other hand, any sum of hermitian squares $\sum_{j}\left|g_{j}(z)\right|^{2} \in \Sigma_{h}$ satisfies the above inequality:

$$
\left|\sum_{j} g_{j}(\alpha) \overline{g_{j}(\beta)}\right|^{2} \leq\left(\sum_{j}\left|g_{j}(\alpha)\right|^{2}\right)\left(\sum_{j}\left|g_{j}(\beta)\right|^{2}\right) .
$$

Since $I \subset J_{\alpha, \beta}$ by assumption, this shows $f \notin \Sigma_{h}+I$.

A more conceptual, and hence simpler, explanation of the above proof is the following. Consider the maximal set $W(I)$ of all points $\alpha \in V(I)$, so that $I \subset J_{\alpha, \beta}$ whenever $\alpha \neq \beta$ and $\alpha, \beta \in W(I)$. Then the polarization of every element $F \in \Sigma_{h}^{2}+I$ defines a positive definite kernel on $W(I) \times W(I)$. Quite specifically, the matrix

$$
\left(F\left(\alpha_{j}, \bar{\alpha}_{k}\right)\right)_{j, k=1}^{m}
$$


is positive semidefinite for every finite subset $\left\{\alpha_{1}, \ldots, \alpha_{m}\right\} \subset W(I)$. On the other hand, positive polynomials along $V(I)$ behave like continuous functions and in particular they do not satisfy in general any matrix positivity, provided that $W(I)$ is nonempty. As mentioned in the introduction, this topic will be exposed in more detail in a forthcoming article dealing with operator positivity aspects of sums of hermitian squares. Similar matrix positivity conditions were recently treated in the article [3].

\section{Examples}

Example 1. We provide an example in two complex variables:

$$
G_{\epsilon}=\left\{(z, w) \in \mathbb{C}^{2} ; \quad \frac{\left|z^{2}-1\right|^{2}}{2}+|w|^{2}+\epsilon\left|\left(z^{2}-1\right) z\right|^{2}<1\right\},
$$

where $\epsilon>0$ is a small parameter. The points $\alpha=(1,1), \beta=(-1,1)$ assure that Proposition 3.1 applies, hence there exist positive polynomials along the boundary of $G_{\epsilon}$ which are not equal to sums of hermitian squares. Indeed, the polarized defining polynomial

$$
P\left(\left(z_{1}, w_{1}\right),\left(\bar{z}_{2}, \bar{w}_{2}\right)\right)=\frac{\left(z_{1}^{2}-1\right)\left(\bar{z}_{2}^{2}-1\right)}{2}+w_{1} \bar{w}_{2}+\epsilon\left(z_{1}^{2}-1\right) z_{1}\left(\bar{z}_{2}^{2}-1\right) \bar{z}_{2}-1
$$

satisfies

$$
P(\alpha, \bar{\alpha})=P(\alpha, \bar{\beta})=P(\beta, \bar{\alpha})=P(\beta, \bar{\beta})=0 .
$$

As a matter of fact, it follows from the proof of Proposition 2.2 referring to the archimedianity of the convex cone $\Sigma_{h}+(P)$ that for large enough $M>0, M-|z|^{2}-|w|^{2}$ is not a sum of hermitian squares modulo the principal ideal $(P)$.

The choice of constants imply that the above inequality defines a connected set with smooth boundary and strictly plurisubharmonic defining function. To check connectedness look at the level sets $|w|=c$, with $0 \leq c \leq 1$. Without the term containing $\epsilon$, equation $\left|z^{2}-1\right|^{2}=2-2 c^{2}$ defines a lemniscate around the foci \pm 1 , which in addition is connected for all $c<1 / \sqrt{2}$. On the other hand, the sections $z= \pm 1$ and $w$ arbitrary connect all level sets $|w|=c$. Again, without the term containing $\epsilon$, a direct computation of the gradient of $P$ proves that the algebraic variety

$$
P_{0}=\frac{\left|z^{2}-1\right|^{2}}{2}+|w|^{2}-1=0
$$

is smooth. Thus, a small additive perturbation of $P_{0}$ by the term $\epsilon\left|\left(z^{2}-1\right) z\right|^{2}$ will not change the smoothness and connectedness of the domain $P<0$. Finally, the hessian matrix of $P$ in complex coordinates is diagonal, with entries $2|z|^{2}+\left|3 z^{2}-1\right|^{2}$ and 1 , hence it is non-degenerate.

This example answers in the negative a question posed by D'Angelo in 2006 at AIM Palo Alto.

Example 2. We depart in this subsection from the several complex variables framework in order to give an example of a domain in $\mathbb{C}$ with compact nonsingular algebraic boundary defined as the level set of a strictly subharmonic polynomial, with the property that on the boundary not every positive polynomial is a sum of hermitian squares. 
Let $a$ be a real parameter, and consider the real hermitian polynomial $p=p_{a}$ in one complex variable $z$, defined by

$$
p_{a}(z, \bar{z})=\left|z^{2}-1+a\right|^{2}+\left|z^{3}-z\right|^{2}-a^{2} .
$$

Since $\partial_{z} \partial_{\bar{z}} p_{a}(z, \bar{z})=4|z|^{2}+\left|3 z^{2}-1\right|^{2}>0$ for $z \in \mathbb{C}$, we see that $p_{a}(z, \bar{z})$ is stricly subharmonic. Below we'll show that, for suitable choice of $a$, the set

$$
\Omega_{a}:=\left\{z \in \mathbb{C}: p_{a}(z, \bar{z})<0\right\}
$$

is non-empty and connected, and the compact real algebraic curve

$$
C_{a}:=\left\{z \in \mathbb{C}: p_{a}(z, \bar{z})=0\right\}
$$

is nonsingular.

On the other hand, from $p_{a}( \pm 1, \pm 1)=0$ (for all four choices of sign) it is obvious that the polarized polynomial $p_{a}(z, w)$ lies in the ideal $J_{1,-1}$. Hence, by Proposition 3.1 , there exists a real polynomial $f \in A$ which is strictly positive on $C_{a}$ but which is not a sum of hermitian squares on $C_{a}$.

Since the polynomials $p_{a}$ form a linear pencil spanned by $p_{0}$ and by $\frac{\partial p}{\partial a}(z, \bar{z})=$ $z^{2}+\bar{z}^{2}-2$, and since the latter defines a nonsingular affine curve, the affine curve $C_{a}$ is nonsingular for all but finitely many choices of $a$. A somewhat tedious calculation by hand, or an immediate check with the help of a computer algebra system, verifies that, for instance, $a=1$ is such a choice.

Moreover, one can show that $\Omega_{a}$ is connected for $a>\frac{1}{2}$. Here we restrict to showing this for $a$ close to 1 . To that end consider $\left|p_{a}\left(r e^{i \theta}\right)\right|^{2}$ as a function of $r \geq 0$, for fixed $\theta$. We find $\left|p_{a}\left(r e^{i \theta}\right)\right|^{2}=h_{a}\left(r^{2}\right)$ where $h_{a}(t)$ is the real cubic polynomial

$$
h_{a}(t)=t^{3}+(1-2 \gamma) t^{2}+(1-2 \gamma+2 a \gamma) t+(1-2 a)
$$

with $\gamma:=\cos (2 \theta)$. If $h_{a}(t)$ has more than one positive root then all three roots are positive, which implies $1-2 \gamma<0$, hence $\gamma>\frac{1}{2}$. On the other hand, the discriminant of $h_{a}(t)$ is negative for $a=1$ and $0 \leq \gamma \leq 1$ (it is equal to $-32 \gamma^{3}+52 \gamma^{2}+8 \gamma-44$ ). Therefore, the discriminant of $h_{a}(t)$ is negative for the same $\gamma$ and all values of $a$ close to 1 . This shows that $h_{a}(t)$ has only one positive root for $a$ close to 1 and all $|\gamma| \leq 1$, which implies that $\Omega_{a}$ is connected for $a$ close to 1 . In summary we have proved:

Proposition 4.1. For all real numbers a close to 1 , the set $\Omega_{a}$ is connected, has a strictly subharmonic defining function and possesses a compact nonsingular algebraic boundary $C_{a}$. There exists a real hermitian polynomial $f(z, \bar{z})$ which is strictly positive on $C_{a}$ but is not a sum of hermitian squares on $C_{a}$.

For $a=1$ we get $\Omega=\left\{z \in \mathbb{C}:|z|^{4}+\left|z^{3}-z\right|^{2}<1\right\}$. Notice that the polynomial $f(z, \bar{z})=2-|z|^{2}$ is strictly positive on $\bar{\Omega}$ but it is not a sum of hermitian squares on $\partial \Omega$, as proved in Proposition 2.2 .

\section{Acknowledgements}

The present note has its origin at a 2006 American Institute of Mathematics (AIM) workshop attended by the first author, and it was completed at a 2009 AIM workshop attended by both authors. They are grateful to AIM for offering an inspiring environment, and they are also indebted to the referee for several constructive comments 
which led to the present improved form of the note. The first author was partially supported by the National Science Foundation Grant DMS 1001071.

\section{References}

[1] J. D'Angelo, Complex variables analogues of Hilbert's seventeenth problem, Int. J. Math. 16(2005), 609-627.

[2] J. D'Angelo, M. Putinar, Polynomial optimization on odd-dimensional spheres, Emerging applications of algebraic geometry, IMA Vol. Math. Appl. 149, Springer, New York, 2009, pp. $1-15$.

[3] J. D'Angelo, D. Varolin, Positivity conditions for Hermitian symmetric functions, Asian J. Math. 7(2003), 1-18.

[4] A. Athavale, Holomorphic kernels and commuting operators, Trans. Am. Math. Soc. 304(1987), 101-110.

[5] J. Bochnak, M. Coste, M-F. Roy, Real Algebraic Geometry. Erg. Math. Grenzgeb. (3) 36, Springer, Berlin, 1998.

[6] D. W. Catlin, J. P. D'Angelo, A stabilization theorem for Hermitian forms and applications to holomorphic mappings, Math. Res. Lett. 3(1996), 149-166.

[7] D. W. Catlin, J. P. D'Angelo, An isometric imbedding theorem for holomorphic bundles, Math. Res. Lett. 6(1999), 43-60.

[8] L. Lempert, Imbedding pseudoconvex domains into a ball, Amer. J. Math. 104(1982), 901-904.

[9] L. Lempert, Imbedding Cauchy-Riemann manifolds into a sphere, Internat. J. Math. 1(1990), 91-108.

[10] E. Løw, Embeddings and proper holomorphic maps of strictly pseudoconvex domains into polydisks and balls, Math. Z. 190(1985), 401-410.

[11] A. Prestel, Ch. N. Delzell, Positive Polynomials. Springer, Berlin, 2001.

[12] M. Putinar, Sur la complexification du problème des moments, C. R. Acad. Sci. Paris, sér. I 314(1992), 743-745.

[13] M. Putinar, Positive polynomials on compact semi-algebraic sets, Indiana Univ. Math. J. 42(1993), 969-984.

[14] D. G. Quillen, On the representation of hermitian forms as sums of squares, Invent. math. 5(1968), 237-242.

[15] C. Scheiderer, Positivity and sums of squares: A guide to recent results, Emerging applications of algebraic geometry, IMA Vol. Math. Appl. 149, Springer, New York, 2009, pp. 271-324.

Department of Mathematics, University of California at Santa Barbara, Santa BarBARA, CAlifornia, 93106-3080

E-mail address: mputinar@math.ucsb.edu

$U R L:$ http://math.ucsb.edu/ mputinar

Fachbereich Mathematik and Statistik, Universität Konstanz, D-78457 Konstanz, GerMANY,

E-mail address: claus.scheiderer@uni-konstanz.de

$U R L$ : http://www.math.uni-konstanz.de/ ${ }^{\text {scheider }}$ 